\title{
Usefulness of the drug allergy passport in the drug allergy management
}

\author{
Olga Branicka, Barbara Rogala, Joanna Glück
}

Department of Internal Diseases, Allergology and Clinical Immunology, Medical University of Silesia, Katowice, Poland

Adv Dermatol Allergol 2021; XXXVIII (3): 433-439

DOI: https://doi.org/10.5114/ada.2021.107930

\begin{abstract}
Introduction: Nowadays, the number of people with drug hypersensitivity has been increasing and it has become a major problem for the healthcare system. Unfortunately, not everyone is aware of which medications they can safely use.

Aim: To assess the suitability of a drug allergy passport in patients with drug hypersensitivity in order to increase knowledge about medicines that can be safely used.

Material and methods: The study was conducted in 54 hospitalized patients with confirmed hypersensitivity to drugs by issuing a drug passport at discharge. The study was carried out with the questionnaire method. The questionnaire was conducted by phone 3, 6 and 12 months after the patients received the drug passport.

Results: Fifty-eight people were contacted by phone. The survey was conducted in 54 people (42 women (77\%), mean age: 48 , range: $19-71$ ), which gives a response rate of $98 \%$. The application of the drug allergy passport by patients increased with time and the number of patients who did not use their passport decreased. With time, patients showed the drug allergy passport to a larger number of doctors, most often to general practitioners and dentists. In the following months, the number of doctors who followed passport recommendations and patients who adhered to the passport recommendations increased.

Conclusions: The analysis of drug allergy passport shows that patients are better informed about medicines they can use and have a greater sense of security. By showing the passport to specialists, they choose the safest and adequate treatment.
\end{abstract}

Key words: drug hypersensitivity reactions, allergy, drug allergy passport.

\section{Introduction}

Currently, the number of people with drug hypersensitivity has been increasing. This is due to high availability of drugs and dietary supplements in pharmacies and other places. Unfortunately, not every patient is aware of which medications he or she can safely use. Drug hypersensitivity reactions (DHR) are the adverse effects of pharmaceutical formulations which clinically resemble allergy and are caused by some immunological mechanism (drug-specific antibody or T-cell) [1]. Clinically the classification includes immediate and non-immediate/delayed DHR. Immediate DHR occurs within 1-6 $\mathrm{h}$ after drug administration and symptoms include urticaria, angioedema, rhinitis, bron- chospasm or anaphylactic shock. Non-immediate DHR occurs at any time later than $1 \mathrm{~h}$ after drug administration. Typical symptoms include maculopapular exanthemas and delayed urticaria [2]. Re-exposure to culprit medications can manifest with severe symptoms or even as a life-threatening reaction. Therefore it is very important to avoid prescribing culprit drugs.

There are different methods of documenting drug allergies. The most popular methods are a written note in paper medical documentation or International Classification of Diseases (ICD-10) code in a computer system. Studies evaluating the effectiveness of those methods do not specify any difference in prescribing errors due to

Address for correspondence: Olga Branicka, Department of Internal Diseases, Allergology and Clinical Immunology, Medical University of Silesia, Katowice, Poland, phone: +48 32789 4641, e-mail: o.branicka@gmail.com Received: 30.10 .2019 , accepted: 14.01 .2020 . 
potentially allergy-inducing drugs, when the allergy was only documented as an ICD-10 code or the information was available in a paper record [3]. However, due to the lack of standardization, none of these methods is sufficiently effective. Therefore, the European Academy of Allergy and Clinical Immunology (EAACI)/European Network of Drug Allergy (ENDA) analysed the situation in Europe and proposed the creation of a common standard allergy passport concerning drugs for patients with drug allergy [4].

According to the nomenclature, DHR is type $B$ of adverse drug reactions (ADR), which is unpredictable, dose-independent and unintended [5, 6]. The current data show that in Europe approximately $3.6 \%$ of all hospital admissions are caused by ADR, and up to $10 \%$ of patients in European hospitals experience an ADR during their hospitalization [7]. When the DHR is caused by several drugs or an uncertain drug, it is recommended to perform the allergological work-up to identify the culprit drug and to select alternative safe drugs [2]. The patient should never be exposed to the culprit drug again, because each subsequent exposure can end with a more serious reaction including life-threatening reactions. Not all patients are able to avoid the culprit drug. Most often this is related to the lack of knowledge of drug allergy history by doctors $[3,8]$. The most important is the first incident when patients reported reactions to primary care physicians. It is important to properly differentiate and refer selected patients for further diagnostics. Then, after the diagnosis, patients should notify other doctors about feedback from the specialist. Often, however, patients forget which drugs they have been prescribed are safe. That is why it is so important to have clear feedback in the form of a drug passport [9].

\section{Aim}

The aim of this study was to assess the usefulness of drug allergy passports for patients with drug allergies and for physicians dealing with them.

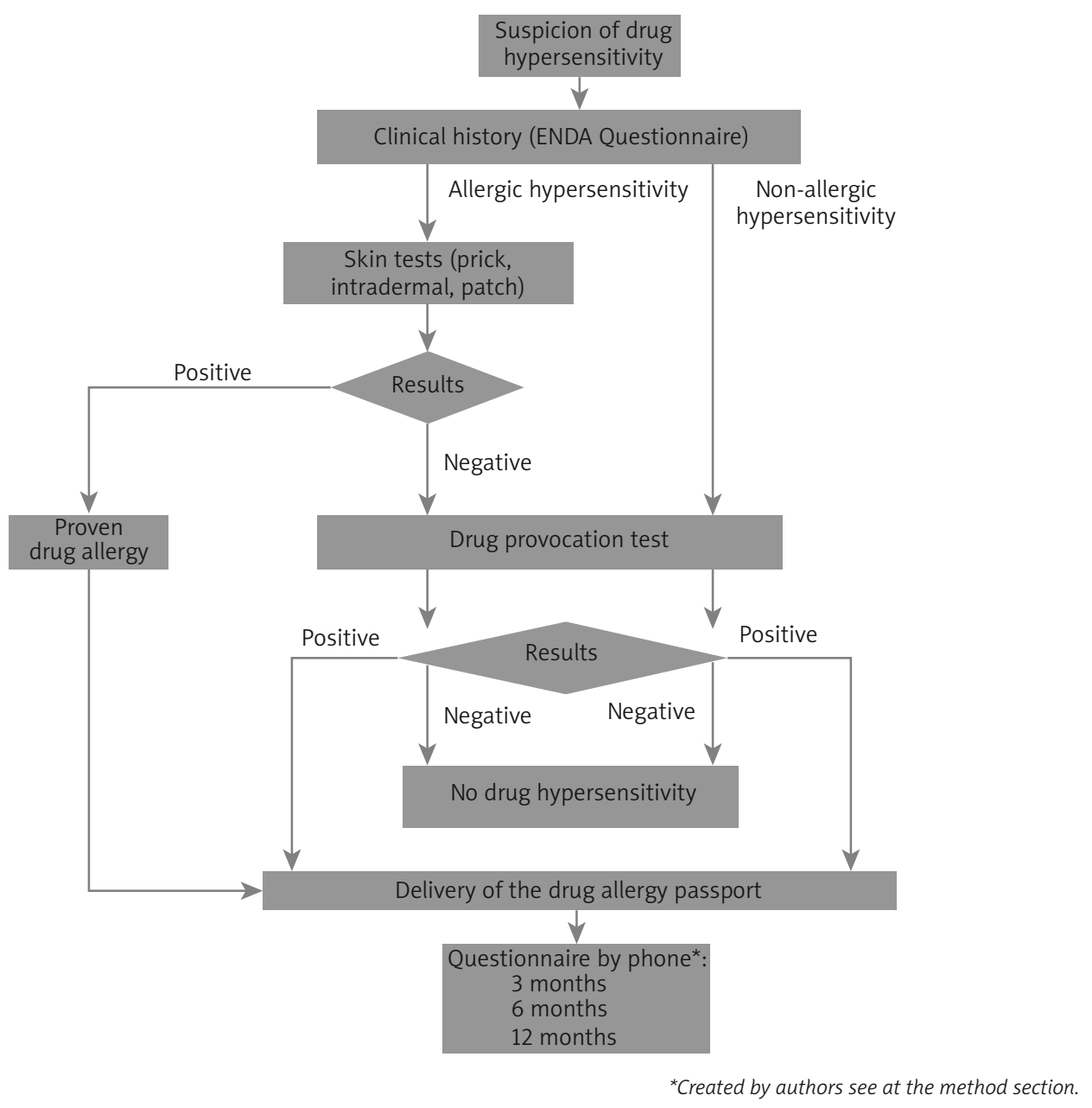

Figure 1. Flow chart of the general diagnostic method in drug hypersensitivity reactions [2, 4] 


\section{Material and methods}

\section{Patient selection}

The study population comprised patients who attended the Department of Allergology from October 2016 to October 2017 with suspected drug allergies. The diagnosis procedure was carried out during routine hospitalization in patients referred with suspected DHR. Patients with drug hypersensitivity confirmed after allergological work-up were included into the study. The study was performed according to the principles of the Declaration of Helsinki. All participants were informed about the study and signed the corresponding informed consent documents to use their personal data for phone contact.

\section{Allergological work-up}

The general rules of diagnostic allergological workup are outlined in Figure 1 and comply with EAACI/ENDA guidelines [2]. DHR diagnostic procedures were carried out 4-6 weeks after complete resolution of all hypersensitivity symptoms during routine hospitalization.

\section{Clinical history}

The clinical history was taken carefully, including symptoms, chronology of symptoms (the interval between administration of the drug and the first symptoms, previous administration), other medications taken and the medical history. To properly record the clinical history we used a questionnaire of ENDA $[4,5]$.

\section{Skin testing}

Patients with hypersensitivity to antibiotics (B-lactams) or local anaesthetics (bupivacaine, lignocaine, mepivacaine) first had skin prick tests (SPT) and then intradermal tests (IDT) done. A precisely defined volume of $0.02 \mathrm{ml}$ was given, causing a bubble with a diameter of $3 \mathrm{~mm}$. The reading performed as a wheal diameter measurement, a positive result is an increase of $>3 \mathrm{~mm}$ with surrounding erythema.

Drug provocation tests (DPT) were performed in the patients' department under the supervision of an expert allergist. DPT was performed with drugs of proved negative skin test reactivity and to find alternative safe drugs. In the case of non-immunological NSAIDs hypersensitivity, non-B-lactams or eye drops, DPTs were performed without previous skin testing. One patient had a suspected delayed allergy to pseudoephedrine. The patch test was done with pseudoephedrine. The reading was taken after 48 and $72 \mathrm{~h}$ [5].

\section{Questionnaire}

Patients with confirmed drug allergy received a drug allergy passport at the end of the hospitalization at discharge. The drug allergy passport was created on the basis of the EAACI and ENDA suggestion [4]. The drug allergy passport comprised patient data, type of drugs which may lead to DHR, methods of confirmation of diagnosis (history, skin testing, DPT), a list of potentially life-threatening drugs and safe alternative drugs with a tolerated dosage. After 3, 6 and 12 months the supervising doctor contacted the patient by telephone to complete the questionnaire. The questionnaire contained 7 questions regarding the following issues: how many times patients used the passport, the presentation of the passports to doctors, following of recommendations by doctors, the use of alternative medicines by patients, the use of medicines which cause DHR, the emergence of drug-related adverse reactions after taking alternative drugs, the patient's assessment of the suitability of the passport (a scale from 1 to 10, where 10-8 means very useful, 7-5 useful, 4-1 not useful) and patients' suggestions of changes in the drug allergy passport (Appendix 1). Patients report only new visits to the specialist, dentist or pharmacist within a specific period of time.

\section{Statistical analysis}

Results are expressed as absolute numbers and percentages. The $\chi^{2}$ test was used to analyse differences in nominal variables between groups. All analyses were performed with a software package (Statistica 12.5). $P$-values less than 0.05 were considered significant.

\section{Results}

Fifty-eight people agreed to be contacted by phone. However, because of technical problems the survey was conducted in 54 patients (42 women, $78 \%$, mean age: 48 years, range: 19-71, 12 men, 22\%, mean age: 49 years, range: 27-66) after 3 months, in 53 patients after 6 months and in 48 patients after 12 months. Types of drugs causing hypersensitivity are shown in Figure 2.

The results of surveys after 3, 6 and 12 months are presented in Table 1.

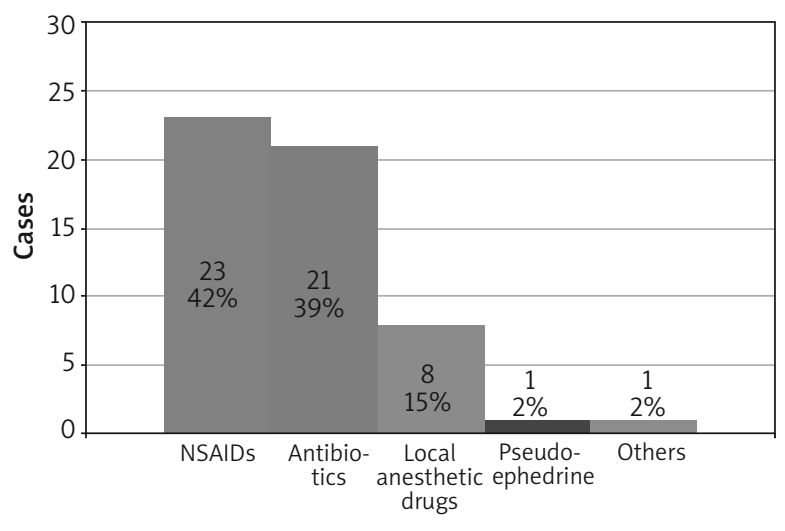

Figure 2. Types of drugs inducing drug hypersensitivity reactions 
Table 1. Results of the questionnaire survey after 3, 6 and 12 months

\begin{tabular}{|c|c|c|c|c|}
\hline \multicolumn{2}{|l|}{ Variable } & \multirow{2}{*}{$\begin{array}{c}\text { After } 3 \text { months } \\
n=54 \\
30(56 \%)\end{array}$} & \multirow{2}{*}{$\begin{array}{c}\text { After } 6 \text { months } \\
\quad n=53 \\
41(77 \%)^{\dagger}\end{array}$} & \multirow{2}{*}{$\begin{array}{c}\text { After } 12 \text { months } \\
n=48 \\
38(81 \%)\end{array}$} \\
\hline Frequency of passport usage & Several times & & & \\
\hline & Once & $14(26 \%)$ & $8(15 \%)$ & $8(17 \%)$ \\
\hline & Never & $10(18 \%)$ & $4(8 \%)^{\ddagger}$ & $1(2 \%)^{\ddagger}$ \\
\hline \multirow{4}{*}{$\begin{array}{l}\text { Kind of health service staff to whom the passport } \\
\text { was shown }\end{array}$} & General practitioner & $30(56 \%)$ & $37(70 \%)$ & $36(77 \%)$ \\
\hline & Specialist & $24(44 \%)$ & $41(77 \%)^{A}$ & $31(66 \%)$ \\
\hline & Dentist & $11(20 \%)$ & $8(15 \%)$ & $8(17 \%)$ \\
\hline & Pharmacist & $4(7 \%)$ & $8(15 \%)$ & $3(6 \%) \ddagger$ \\
\hline \multicolumn{2}{|c|}{ Number of patients who used the alternative drugs on their own $n(\%)$} & $30(55 \%)$ & $14(26 \%)^{\dagger}$ & $19(40 \%)$ \\
\hline \multicolumn{2}{|c|}{ Number of doctors who prescribe the alternative drugs on their own $n(\%)$} & $40(74 \%)$ & $46(87 \%)$ & $46(96 \%)^{c}$ \\
\hline \multicolumn{2}{|c|}{$\begin{array}{l}\text { Number of patients who did not take medicines that potentially cause drug } \\
\text { hypersensitivity reactions }\end{array}$} & $54(100 \%)$ & $53(100 \%)$ & $48(100 \%)$ \\
\hline \multirow[t]{3}{*}{ Passport usefulness rate in patients' opinion } & Not useful (4-1) & $1(2 \%)$ & 0 & 0 \\
\hline & Useful (7-5) & $1(2 \%)$ & $2(6 \%)^{\ddagger}$ & 0 \\
\hline & Very useful (10-8) & 52 (96\%), & $51(94 \%)$ & 48 (100\%) \\
\hline
\end{tabular}

${ }^{t} p=0.02,{ }^{\ddagger} p=0.0001,{ }^{A} p=0.0005,{ }^{c} p=0.002$.

The application of the drug allergy passport by patients increased with time and the number of patients who did not use their passport decreased. At 6 months significantly more patients answered that they used the passport several times and it was stable also at 12 months. Accordingly, the number of patients who answered "never" significantly decreased at 6 and 12 months. With time, patients showed the drug allergy passport to a significantly larger number of specialists (at 6 months), however the number of general practitioners and dentists to whom the passport was shown was stable. The number of pharmacists who saw the passports was relatively low as compared to doctors, and significantly decreased at 12 months as compared to that at 6 months.

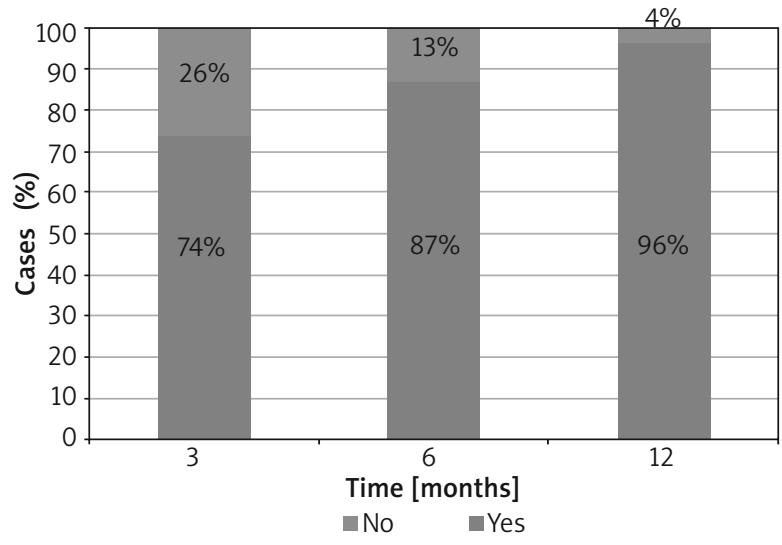

Figure 3. Alternative drugs prescribed by the doctors based on the drug allergy passport (in all comparisons, $\chi^{2}$ test, $p>0.05$ )
The number of doctors who prescribed alternative drugs was stable at 6 months and significantly increased at 12 months as compared to that at 3 months (Figure 3).

The number of patients who used alternative drugs on their own was comparable at 3 and 12 months, but was significantly lower than that at 6 months.

The hypersensitivity reaction after selected alternative medicines occurred only in 2 (4\%) patients during the first 3 months of follow-up.

The proposed form of the passport was acceptable by all patients. The only suggestions were technical as some patients suggested a smaller version of the passport like an ID card or bracelet.

\section{Discussion}

In this study, we conducted a prospective survey in which we demonstrated the usefulness of the drug allergy passport in patients with DHR. Patients who received the drug allergy passport and had selected safe alternative drugs showed it to a general practitioner, to a specialist, to a dentist and even to a pharmacist. This facilitated the work of doctors dealing with the patients with DHR. Doctors adhering to the recommendations of the passport did not prescribe drugs that were classified as culprits. Patients avoided re-contact and reactions that could be potentially life-threatening.

The EAACI task force report on management of drug hypersensitivity in primary care demonstrates that only $38.5 \%$ of primary care physicians felt reasonably competent in DHR diagnosis and 63\% expressed a high or medium need for further education $[9,10]$. In many cases the primary care physician will label the majority of patients 
as allergic without further enquiry, leading to problems related to overdiagnosis. It is very important that primary care physicians can correctly recognize type $B$ reactions and, if appropriate, refer patients to specialists for further diagnosis. Patients with DHR after diagnosis should always carry information about culprit drugs with them and should share it with all healthcare professionals. Analysis of the results of our questionnaire study showed that no patients which had the drug allergy passport took medicines that potentially caused drug hypersensitivity reactions. By showing the passport to doctors a safer therapy was chosen [11-13]. In some countries, there are electronic health records (EHRs) with a special "allergy" list containing other types of adverse drug events (ADE), such as drug intolerance, drug toxicity, drug idiosyncrasy and pseudoallergic reactions. Such information allows physicians of various specializations dealing with the patient to know the medicines the patient is sensitive to. In addition, they are a valuable source of information on the incidence and frequency of DHR with regard to specific drug groups [14]. Information on the patient's disease is also used in other disease entities and not only allergic. The most popular form is wearing bracelets with information about diabetes, epilepsy, haemophilia, implanted pacemaker or cardioverter defibrillator. Patients also usually have small books, leaflets or passports with more details about treatment and the centre in which the treatment is taking place. It is a very easy and clear way to quickly help in case of need. In some cases, the initiative to "distinguish" the patient and to have exhaustive information about the disease comes from the patients themselves creating a community. In the field of allergy, hereditary angioedema, there is a very active patient community. They provide training to patients on coping with exacerbations and increasing the awareness of the disease.

In this study, we observed only 2 cases of drug allergies with alternative drugs, although those patients were equipped with the passport. The first case was a 68-year-old woman suffering from glaucoma and using eye drops to reduce intraocular pressure. Despite the negative tolerance test of alternative eye drops she used, eyelid oedema, lacrimation and burning sensation appeared in subsequent applications. It was probably caused by allergy to conservatives, and not to an active drug. The other case was a 32-year-old woman with an allergy to B-lactam antibiotics. We found negative tolerance tests with alternative drugs, namely ciprofloxacin, roxithromycin and doxycycline. However, a subsequent use of ciprofloxacin for the urinary tract infection caused urticaria, probably resulting from direct mast cell degranulation reactions.

In the present study, a very positive assessment of the drug allergy passport use by patients in their daily lives was found. The patients find the passport clear and easy to use, regardless of their age and level of education. In addition, patients who carried it in a purse or wallet felt safer. Due to the presentation of the drug allergy passport to medical staff the patients did not have to remember the difficult names of potentially life-threatening drugs. Currently, there is a mobile phone application called "drug allergy" available in some medical centres. Unfortunately, for most older patients or patients who do not have a smartphone, this application is not useful due to limited knowledge of how to use it. In medical practice, passports are used in different conditions. For example, a Healthcare Passport has been started to be used in older people with dementia. It may improve the communication with a range of health and social support services. It contains a compendium of information about the person with dementia (not only family members, activities, interests but also information about diagnoses, medications and other treatments) [15]. "My Medication Passport" (MMP) developed by the National Institute for Health Research was used by elderly patients and contained a list of all medications. Then the application of MMP was extended to a paediatric version for children with multiple disabilities. MMP helps children and young people, parents and caregivers to manage medicines more effectively [16].

There are some limitations of this study. It may be biased by problems with patients remembering when and how many doctors they visited and what kind of drugs they used after 6 or 12 months. Therefore, patients may not always provide accurate data. It may result in some differences among data at 3, 6 and 12 months which theoretically should accumulate.

The initiative of ENDA is not a first version of passports in disease management but it is of significant importance because it helps patients to understand the problem of DHR and shows how to avoid serious consequences associated with re-administration of potentially dangerous medicines.

\section{Conclusions}

The findings of this study emphasize the necessity of delivering the drug allergy passport to patients and confirm that they are better informed about the safe medicines. The patients and their families are more aware of the drug or drug classes they need to avoid. The drug allergy passport makes physicians aware of patients' drug allergies when they prescribe medicines and permit them to choose the safest treatment and adequate therapy and avoid dangerous drugs.

In conclusion, as drug allergies are considered the main and often neglected risk of pharmacological therapy, the awareness of patients and physicians should be increased to avoid repeated administration of dangerous substances.

\section{Conflict of interest}

The authors declare no conflict of interest. 


\section{References}

1. Johansson SG, Bieber T, Dahl R, et al. Revised nomenclature for allergy for global use: Report of the Nomenclature Review Committee of the World Allergy Organization, October 2003. J Allergy Clin Immunol 2004; 113: 832-6.

2. Demoly P, Adkinson NF, Brockow K, et al. International Consensus on drug allergy. Allergy 2014; 69: 420-37.

3. Benkhaial A, Kaltschmidt J, Weisshaar E, et al. Prescribing errors in patients with documented drug allergies: comparison of ICD-10 coding and written patient notes. Pharm World Sci 2009; 31: 464-72.

4. Brockow K, Aberer W, Atanaskovic-Markovic M, et al. Drug allergy passport and other documentation for patients with drug hypersensitivity - an ENDA/EAACI Drug Allergy Interest Group Position Paper. Allergy 2016; 71: 1533-9.

5. Brockow K, Romano A, Blanca M, et al. General considerations for skin test procedures in the diagnosis of drug hypersensitivity. Allergy 2002; 57: 45-51.

6. Edwards IR, Aronson JK. Adverse drug reactions: definitions, diagnosis, and management. Lancet 2000; 356: 1255-9.

7. Bouvy JC, De Bruin ML, Koopmanschap MA. Epidemiology of adverse drug reactions in Europe: a review of recent observational studies. Drug Saf 2015; 38: 437-53.

8. Buhl T, Meynberg HC, Kaune KM, et al. Long-term follow-up of patients with hypersensitivity to nonsteroidal anti-inflammatory drugs reveals shortcomings in compliance and care. J Allergy Clin Immunol 2011; 127: 284-5.

9. Doňa I, Caubet JC, Brockow K, et al. An EAACl task force report: recognising the potential of the primary care physician in the diagnosis and management of drug hypersensitivity. Clin Transl Allergy 2018; 8: 16.

10. Ryan D, Angier E, Gomez M, et al. Results of an allergy educational needs questionnaire for primary care. Allergy 2017; 72: 1123-8.

11. Pérez-Alzate D, Cornejo-Garcia JA, Pérez-Sánchez N, et al. Immediate reactions to more than 1 NSAID must not be considered cross-hypersensitivity unless tolerance to ASA is verified. J Investing Allergol Clin Immunol 2017; 27: 32-9.

12. Calvo Campoverde K, Giner-Muńoz MT, Martínez Valdez L, et al. Hypersensitivity reactions to non-steroidal anti-inflammatory drugs and tolerance to alternative drugs. An Pediatr (Barc) 2016; 84: 148-53.

13. Buonomo A, Pascolini L, Rizzi A, et al. Cross-reactivity and tolerability of ertapenem in patients with IgE-mediated hypersensitivity to $\beta$-lactams. J Investig Allergol Clin Immunol 2016; 26: 100-10.

14. Zhou L, Dhopeshwarkar N, Blumenthal KG, et al. Drug allergies documented in electronic health records of a large healthcare system. Allergy 2016; 71: 1305-13.

15. Leavey G, Abbot A, Watson M, et al. The evaluation of a healthcare passport to improve quality of care and communication for people living with dementia (EQuIP): a protocol paper for a qualitative, longitudinal study. BMC Health Serv Res 2016; 16: 363.

16. Jubraj B, Blair M. Use of medication passport in a disable child seen across many care settings. BMJ Case Rep 2015; 2015: bcr2014208033. 
Appendix 1. Questionnaire for patients with drug allergy passport

Questionnaire for patients with the "Drug Allergy Passport"

Name of the patient

Date of delivery of the drug allergy passport

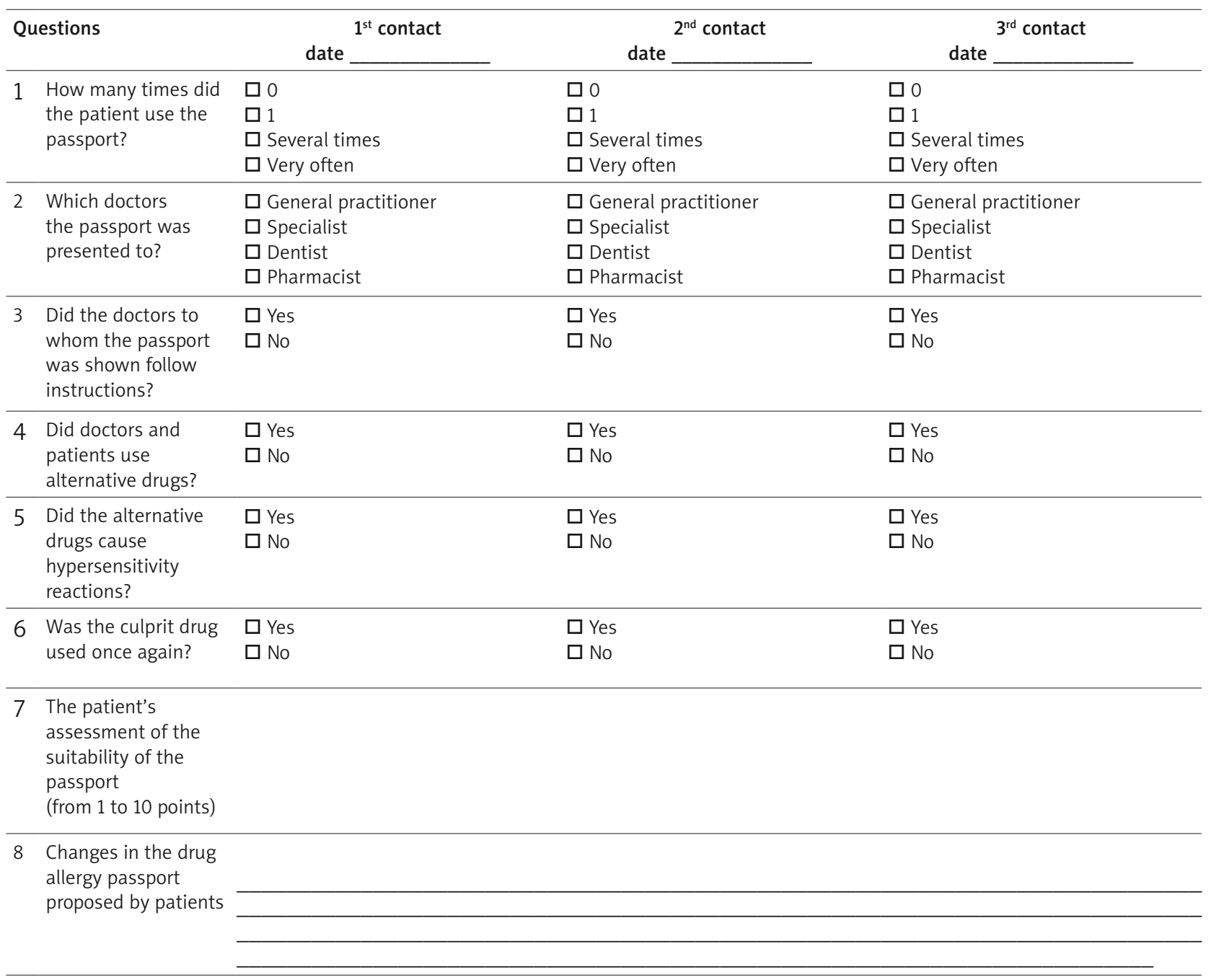

